22. Rivard, C. and Vigneault, H., Trend detection in hydrological series: when series are negatively correlated. Hydrol. Process., 2009, 23(19), 2737-2743.

23. Yue, S., Pilon, P., Phinney, B. and Cavadias, G., The influence of autocorrelation on the ability to detect trend in hydrological series. Hydrol. Process., 2002, 16(9), 1807-1829.

24. Durbin, J. and Watson, G. S., Testing for serial correlation in least squares regression. I. Biometrika, 1950, 37(3/4), 409-428.

25. Durbin, J. and Watson, G. S., Testing for serial correlation in least squares regression. II. Biometrika, 1951, 38(1/2), 159-177.

26. Durbin, J. and Watson, G. S., Testing for serial correlation in least squares regression. III. Biometrika, 1971, 58(1), 1-19.

27. Naidu, C. V., Durgalakshmi, K., Krishna, K. M., Rao, S. R., Satyanarayana, G. C., Lakshminarayana, P. and Rao, L. M., Is summer monsoon rainfall decreasing over India in the global warming era? J. Geophys. Res., 2009, 114(D24), 1-16.

28. Malik, N., Bookhagen, B. and Mucha, P. J., Spatiotemporal patterns and trends of Indian monsoonal rainfall extremes. Geophys. Res. Lett., 2016, 43(4), 1710-1717.

29. Paul, S., Ghosh, S., Oglesby, R., Pathak, A., Chandrasekharan, A. and Ramsankaran, R. A. A. J., Weakening of Indian summer monsoon rainfall due to changes in land use land cover. Sci. Rep., 2016, 6, 32177; doi:10.1038/srep32177.

ACKNOWLEDGEMENTS. I thank Mr Kamakhya Bose (Office of Deputy Director of Agriculture (Administration), Maldah district, Government of West Bengal) for providing the necessary climatic data. I also thank Mr Martin Jakob (Institute of Cell Biology, University Bern, Switzerland) and Mr Jason Michael Whyte (University of Melbourne, Australia) for assistance.

Received 19 September 2018; revised accepted 25 November 2019

doi: $10.18520 / \mathrm{cs} / \mathrm{v} 119 / \mathrm{i} 6 / 1031-1038$

\section{Food availability rhythm affects reproduction in consecutive generations of ladybird, Propylea dissecta}

\author{
Arshi Siddiqui ${ }^{1,2}$, Omkar $^{1}$ and \\ Geetanjali Mishra ${ }^{1, *}$ \\ ${ }^{1}$ Ladybird Research Laboratory, Department of Zoology, \\ University of Lucknow, Lucknow 226 007, India \\ ${ }^{2}$ Department of Bioscience, Integral University, Dasauli Kursi Road, \\ Lucknow 226 026, India
}

Transgenerational effect on developmental attributes of insects has been studied extensively. The present study elucidates the role of diet on transgenerational developmental duration and reproductive attributes of

*For correspondence. (e-mail: geetanjalimishra@hotmail.com)

1038
Propylea dissecta (Mulsant). We have found a significant direct and transgenerational role of diet variations in feeding rhythms on the above attributes of $P$. dissecta. Dampened response was found in offspring fed on intermittent diet even when their parental generation was provided abundant food supply. Reduced reproductive attributes were found in the progeny of abundant diet parents reared on intermittent diet than the kin of the same abundant fed parents which were under other diet conditions.

Keywords: Feeding behaviour, ladybirds, pea aphid, stress, transgenerational effect.

THE environment affects reproduction as well as development of an individual directly and/or indirectly. Direct effect on development and reproduction of multiple taxa including insects are mediated through resource availability, resource quality, climate variations ${ }^{1}$, and many other environmental factors ${ }^{2}$. When environment affects the parents and influences the development and reproduction of their progeny, indirect environmental effects are seen. Often, this is known to occur through parental exposure and experience $\mathrm{e}^{3,4}$. The nutritional environment of the parental generation can probably influence the developmental and reproductive potential of the next generation through food-based maternal effects ${ }^{5}$. Diet manipulations are possibly associated with increased longevity as well as stress tolerance, ${ }^{6,7}$ in the offspring of starved diet parents; for example, as in ladybird Anegleis cardoni (Weise) $)^{5}$.

Reynolds et $a l .{ }^{8}$ reported that most insects feed in bouts and have varying inter-feed gap lengths. Also, the feeding period length of many insects is strongly correlated to the preceding inter-feed gap duration'. Food deprivation is compensated by controlling the total percentage of feeding time by adjusting feed bout length or inter-feed gap length.

Studies have shown volumetric feedback response to regulate meal length variability through gut stretch receptors $^{10,11}$. There are other mechanisms between feeding extremes that indicate patterns and trends extending from minutes to hours. Previous studies also indicate that rhythmic cycles are common in biological environments, ranging from very fast biochemical alternations of seconds or milliseconds inside cells to circadian rhythms of individual organisms ${ }^{12}$. Thus, we hypothesized the following in the present study in two consecutive generations: (1) whether responses were dependent on the frequency of food deprivation, and (2) whether the effect of food deprivation on developmental plasticity is transferred to the offspring through parental effect. Thus, the main aim in the transgenerational assessments was to evaluate whether progeny from starved parents were inclined to cope with the lack of food by parental perceptions. 
Adults of Propylea dissecta (Mulsant) were collected from aphid-infested agricultural fields near Lucknow, Uttar Pradesh, India, to establish a stock culture. Mating pairs of adults were kept in clear plastic petri dishes $(9.0 \times 2.0 \mathrm{~cm})$ with pea aphids, Acyrthosiphon pisum (Harris) (Hemiptera: Aphididae) reared on Vicia faba L. (Fabaceae) (broad-bean cultures were maintained at $21^{\circ} \pm 1{ }^{\circ} \mathrm{C} ; 65 \pm 5 \%$ relative humidity $(\mathrm{RH})$ in a glasshouse). Paired adults were kept in a BOD incubator (YORCO; York Scientific Industries Pvt Ltd, India) maintained at $27^{\circ} \pm 1^{\circ} \mathrm{C}$ temperature; $65 \pm 5 \% \quad \mathrm{RH}$ and $14 \mathrm{~L}: 10 \mathrm{D}$ photoperiod. The eggs laid were separated and aphid prey supplies to adults were replenished every $24 \mathrm{~h}$. Adaptation of ladybirds to laboratory conditions was ensured by maintaining the stock for four generations prior to experimentation. Wild stock was continually introduced to the breeding stock to prevent inbreeding. Neonates were maintained individually at the aforementioned laboratory conditions and experiments were performed using the required developmental stages.

Two group of studies were planned to evaluate: (i) immediate (parental generation) and (ii) transgenerational (progeny generation) response of food deprivation in two successive generations.

Early third instar larvae ( $n=20$ per treatment) were individually put in plastic petri dishes under any one of the following conditions: (i) food deprivation for $0 \mathrm{~h}$ (control = no starvation); (ii) food deprivation for $24 \mathrm{~h}$; (iii) food deprivation for $48 \mathrm{~h}$; (iv) food deprivation for $72 \mathrm{~h}$, and (v) food deprivation for $96 \mathrm{~h}$, so as to standardize starvation durations for further experimentation. No third instars subjected to 72 and $96 \mathrm{~h}$ food deprivation survived. Third instar larvae were selected for starvation standardization as these are sturdier than the early instar and likely to survive stress better, and also because they are more voracious. Also, exposing third instars to treatments allowed a longer duration of exposure than would be possible if fourth instars were used.

Based on starvation standardization results in third instars, 500 randomly selected early (prior to sclerotization post moulting) third instar larvae were individually put in plastic petri dishes and provided either (i) ad libitum prey supply till pupation (abundant, A); (ii) ad libitum prey supply for $24 \mathrm{~h}$ followed by $12 \mathrm{~h}$ starvation (intermittent, I), or (iii) $12 \mathrm{~h} \mathrm{ad} \mathrm{libitum} \mathrm{prey} \mathrm{supply} \mathrm{followed} \mathrm{by} 12 \mathrm{~h}$ starvation (sub-intermittent, S). All treatments were continued until pupation. On emergence, adult mass was taken $2 \mathrm{~h}$ after emergence and following that, they were reared on ad libitum prey until they were 10-days-old.

These 10-day-old adults, belonging to each of the three larval food regimens, were allowed to mate once after which the females were separated and maintained on ad libitum feed. Oviposition and per cent egg viability were documented over the next five days, and survival of adults was also recorded.
To determine the role of parental larval diet on lifehistory attributes of offspring, we randomly selected offspring (200 neonates from each parental diet regime, i.e. A, I and S) from all three treatments of parental diet experiments. These were reared separately in plastic petri dishes and provided ad libitum prey till they reached third instar stage. These third instars of each parent diet were separately placed in plastic petri dishes and then put in any one of the three groups, viz. A, I or S. Earlier instars were not exposed to these treatments as they were unable to sustain them. The experimental set-up thus consisted of the following treatment groups, viz. (1) parental abundant, larval abundant (AA), (2) parental abundant, larval intermittent (AI), (3) parental abundant, larval subintermittent (AS), (4) parental intermittent, larval abundant (IA), (5) parental intermittent, larval intermittent (II), (6) parental intermittent, larval sub-intermittent (IS), (7) parental sub-intermittent, larval abundant (SA), (8) parental sub-intermittent, larval intermittent (SI), and (9) parental sub-intermittent, larval sub-intermittent (SS). Once adults emerged from these treatments, they were separated and given ad libitum food. Mating pairs were formed within the treatments when the adults were 10days-old. Oviposition of isolated females and per cent egg viability were observed for five days just after mating, and survival of adults was also recorded.

Statistical significance of the data on partial developmental duration, adult body mass as well as reproductive attributes (which include fecundity and per cent egg viability) of parental generation was determined by one-way ANOVA followed by post-hoc Tukey's honest test of significance at 5\% levels; variation in starvation regime was considered as the independent factor.

To analyse the combined effect of diet of parent and progeny generations, data on partial developmental duration, adult body mass as well as reproductive attributes (fecundity and per cent egg viability) of progeny generation, were subjected to two-way ANOVA with diets of parent and progeny generations acting as independent factors. All analyses were done using MINITAB 15.0.

Results of one-way ANOVA revealed that both the partial developmental duration and adult body mass of parent generation adults were significantly influenced by the diet regime provided to larvae (Table 1). Both reproductive parameters (fecundity and \% egg viability) were highest when they had been fed on an abundant diet; however, the lowest reproductive output was on intermittent diet.

Two-way ANOVA revealed that partial developmental duration was affected by parental as well as progeny diets; and also revealed their significant interaction (Table 1). On various sub-diet regimes, the progeny generation instars took the longest duration to develop on intermittent diet while those maintained on abundant diet regime took the shortest duration to complete development. The results revealed that the development of 


\section{RESEARCH COMMUNICATIONS}

Table 1. ANOVA showing the effect of diets on partial developmental duration, adult body mass and fecundity and per cent egg viability in both parent as well as progeny generations respectively

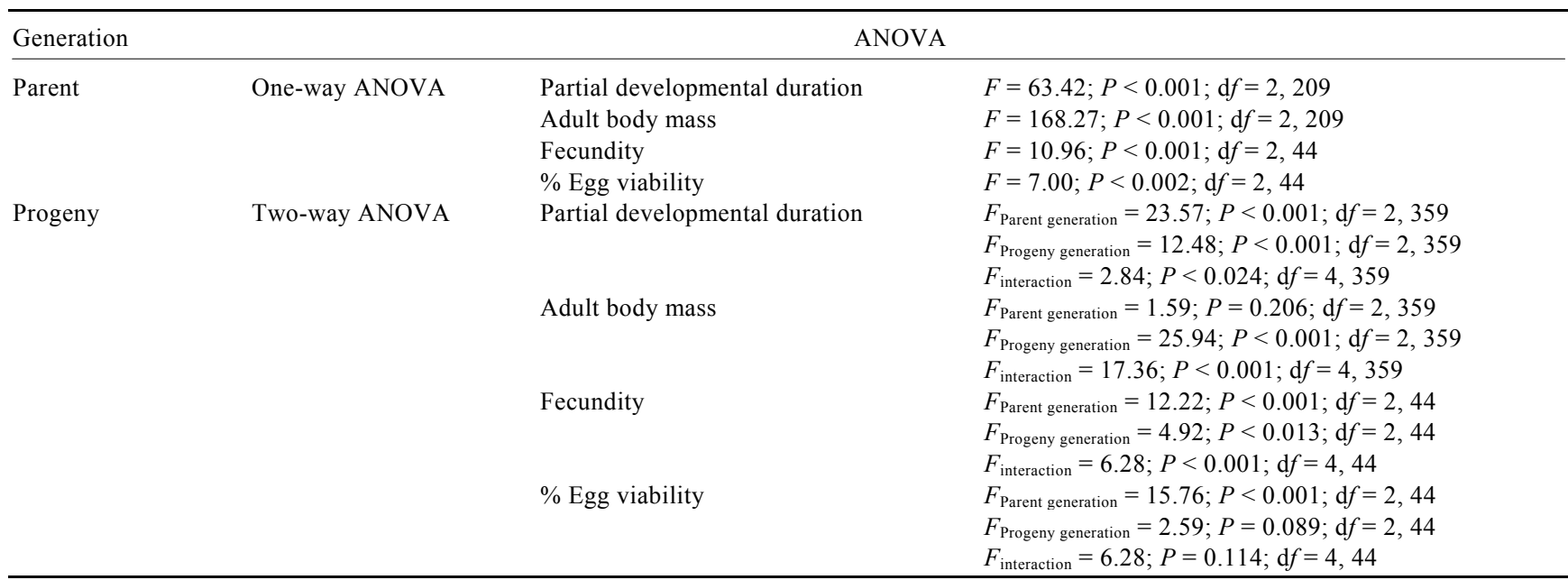

immature stages of the progeny generation was quickest on abundant (AA) and slowest on intermittent (II) feeding regimes, regardless of the diet provided to the previous generation. Similar result was also observed for adult body weight. Progeny of parents fed with abundant diet exhibited higher per cent egg viability and fecundity than those that had been provided with intermittent or subintermittent diet. However, progeny of parents reared on intermittent diet laid significantly higher number of eggs on intermittent diet than on abundant and sub-intermittent diet. Also, progeny of parents reared on sub-intermittent diet fared better on the same diet than on intermittent diet, and almost at par on abundant diet. Per cent egg viability also showed similar trends.

Diet, parental as well as of progeny, was found to influence fecundity significantly, while no such effect was seen on per cent egg viability regardless of parental diet (Table 1).

Our findings reveal strong transgenerational impact of food deprivation on development and reproduction of ladybird beetles. Parent experiment revealed that the fluctuating diet affects the development and survival of stages experiencing food deprivation. We studied the transgenerational effect of diet fluctuations (inter-feed) on development and reproductive attributes of progeny of the parent generation.

Ladybird larvae are more voracious and efficient in catching aphids as they grow in size, with the fourth instar larvae taking $40 \%$ of the overall growth period and eating $65 \%$ of the total food required to complete development ${ }^{13}$. Fluctuations in the larval diet of parental generation influenced fecundity and per cent egg viability. As expected, development was rapid under abundant conditions rather than the two fluctuating interrupted diet regimes. Fecundity in particular may be influenced by intermittent food, as it may lead to delayed development of egg chorion ${ }^{14}$. Loss in sperm production may be respon- sible for reduced egg viability ${ }^{15}$. It was interesting to note that in the parent generation, fecundity was higher in the sub-intermittent fluctuation and not in the intermittent one as expected, probably owing to more duration of food availability than the former. It is likely that this could be due to the lack of synchronicity of the intermittent regime with the innate circadian rhythm of the used. The intermittent regime apparently provides more ambiguous knowledge on the availability of food, and is also out of step with the intrinsic circadian rhythm. Conversely, subintermittent diet in which the feeding pattern was equally distributed in $12 \mathrm{~h}$ oscillation, resulted in a more predictable regime for the circadian rhythm of the insect. Food quantity greatly influences the intrinsic growth and reproductive rate of ladybirds ${ }^{16}$.

In the progeny generation, fluctuations of the preceding generation were reinforced. While it was expected that those provided abundant conditions would perform better regardless of the experience of the parent generation, this was found to be predominant when even the parent generation experienced abundant conditions. Adults with experience of sub-intermittent exposures in parent and progeny generations performed reproductively better or at par with adults that had a parent generation with sub-intermittent and a progeny generation with abundant diet.

The reduced egg viability under intermittent and subintermittent fluctuating diet regimes may be due to variable length of non-feeding durations followed by short feeding duration. Interestingly, progeny of abundant diet parent provided with abundant intermittent (AI) diet showed minimum fecundity with maximum egg viability than those provided with intermittent and sub-intermittent diets respectively. This is possibly attributable to costbenefit scenario where there is more egg with less viability or vice versa, hence low number of eggs with more viability substance and viability likelihood would be a gain 
in present cases. Studies also revealed that the scarcity of food influences fitness of the progeny ${ }^{17}$.

Similar results, though not as prominent, were observed for development of adult body mass in parent and progeny generations. These can be attributed to the same lack or presence of synchronicity with the innate circadian rhythm, which resulted in stress alleviating or stress inducing factors. These effects could be transmitted to the developing offspring during various stages and in a variety of ways. Simply, the development of immature stages could be constrained directly or indirectly. For example, parents on intermittent and sub-intermittent dietary conditions produce low-quality offspring ${ }^{18}$. Nevertheless, some contradictions in current research have shown that nutrient shortage in parental diet was not made up entirely by providing sufficient diet to the offspring; as in sooty copper Lycaena tityrus Poda $^{19}$ and Telostylinus angusticollis (Enderlein) (Diptera: Neriidae) ${ }^{4}$.

The influence of diets of prior generations is similar to that reported by Vijendravarma et al. ${ }^{20}$, in that maternal nutritional effects tend to have both beneficial and maladaptive impacts on the success of the offspring. The present findings thus provide evidence of within and transgenerational evolutionary mechanisms underlying strategies of developmental variants during unfavourable conditions. This study concludes that some kind of circadian rhythm for feeding is present, that possibly exists for feeding or non-feed durations in animals. Further experiments are necessary on the probable transgenerational effects in $P$. dissecta.

\section{Competing interests: None.}

1. Houghton, J. T. et al., Climate Change 2001: The Scientific Basis, Cambridge University Press, Cambridge, UK, 2001.

2. Gilbert, S. F., Mechanisms for the environmental regulation of gene expression: ecological aspects of animal development. $J$. Biosci., 2005, 30, 65-74.

3. Bonduriansky, R. A. and Head, M., Maternal and paternal condition effects on offspring phenotype in Telostylinus angusticollis (Diptera: Neriidae). J. Evol. Biol., 2007, 20, 2379-2388.

4. Mousseau, T. A. and Fox, C. W., The adaptive significance of maternal effects. Trends Ecol. Evol., 1998, 13, 403-407.

5. Omkar, Kumar, G. and Sahu, J., Performance of a predatory ladybird beetle, Anegleis cardoni (Weise) (Coleoptera: Coccinellidae) on three aphid species. Eur. J. Entomol., 2009, 106, 565-572.

6. Bubli, O. A., Imasheva, A. G. and Loeschcke, V., Selection for knockdown resistance to heat in Drosophila melanogaster at high and low larval diet. Evolution, 1998, 52, 619-625.

7. Smith, E. M. et al., Feeding Drosophila a biotin-deficient diet for multiple generations increases stress resistance and lifespan and alters gene expression and histone biotinylation patterns. J. Nutr., 2007, 137, 2006-2012.

8. Reynolds, S. E., Yeomans, M. R. and Timmins, W. A., The feeding behaviour of caterpillars (Manduca sexta) on tobacco and on artificial diet. Physiol. Entomol., 1986, 11, 39-51.

9. Blaney, W. M., Chapman, R. F. and Wilson, A., The pattern of feeding of Locusta migratoria (L.) (Orthoptera: Acrididae). Acrida, 1973, 2, 119-137.
10. Simpsons, S. J., The role of volumetric feedback from the hindgut in the regulation of meal size in fifth instar Locusta migratoria nymphs. Physiol. Entomol., 1983, 8, 451-467.

11. Simpson, S. J. and Simpson, C. L., The mechanisms of compensation by phytophagous insects. In Insect-Plant Interactions (ed. Bernays, E. A.), CRC Press, Boca Raton, Fla., USA, 1989, vol. 2, pp. $112-160$.

12. Bernays, E. A. and Singer, M. S., A rhythm underlying feeding behaviour in a highly polyphagous caterpillar. Physiol. Entomol., 1998, 23, 295-302.

13. Hodek, I., Van Emden, H. F. and Honek, A., Ecology and Behaviour of the Ladybird Beetles (Coccinellidae), John Wiley Ltd, UK, 2012, p. 4229.

14. Lemos, W. P., Medeiros, R. S., Ramalho, F. S. and Zanuncio, J. C., Effects of plant feeding on the development, survival and reproduction of Podisus nigrispinus (Dallas) (Heteroptera: Pentatomidae). J. Integr. Pest Manage., 2001, 47(2), 89-93.

15. Droney, D. C., Environmental influences on male courtship and implications for female choice in a lekking Hawaiian Drosophila. Anim. Behav., 1996, 51, 821-830.

16. Lawo, J. P. and Lawo, N. C., Misconceptions about the comparison of intrinsic rates of natural increase. J. Appl. Entomol., 2011, 135, 715-725.

17. Agarwala, B. K. and Bhowmik, A. K., Effect of resource gradient on age and size at maturity and their influence on early-life fecundity in the predatory Asian lady beetle, Harmonia axyridis. Entomol. Exp. Appl., 2011, 141, 97-102.

18. Omkar, Sahu, J. and Kumar, G., Effect of prey quantity in a ladybird beetle, Anegleis cardoni (Weise) (Coleoptera: Coccinellidae). Int. J. Trop. Insect. Sci., 2010, 30(1), 48-56.

19. Fischer, K. and Fiedle, K., Sexual differences in life-history traits in the butterfly Lycaena tityrus: a comparison between direct and diapause development. Entomol. Exp. Appl., 2001, 100(3), 325330 .

20. Vijendravarma, R. K., Narasimha, S. and Kawecki, T. J., Effects of parental diet on egg size and offspring traits in Drosophila. Biol. Lett., 2010, 6, 238-241.

ACKNOWLEDGEMENTS. A.S. and G.M. thank the Department of Science and Technology, New Delhi for financial assistance under the Fast Track Young Scientist Scheme. Omkar thanks the Department of Higher Education, Government of Uttar Pradesh, India for providing assistance under the Centre of Excellence programme.

Received 19 March 2020; revised accepted 24 June 2020

doi: $10.18520 / \mathrm{cs} / \mathrm{v} 119 / \mathrm{i} 6 / 1038-1041$ 